\title{
The prevalence of upper respiratory symptoms in a cohort of adults presenting with symptoms of gastro-oesophageal reflux disease
}

\author{
D L Amarasiri ${ }^{1}$, A Pathmeswaran ${ }^{2}$, A S Dassanayake ${ }^{3}$, A P de Silva ${ }^{4}$, M D B Adikari ${ }^{1}$, P A B Sanjeewa ${ }^{1}$, \\ A Jayaratne $^{1}$, H J De Silva ${ }^{4}$
}

(Index words: upper respiratory symptoms, gastro-oesophageal reflux, GORD)

\begin{abstract}
Introduction Gastro-oesophageal reflux disease (GORD) is the pathological reflux of gastric contents into the oesophagus. The oesophagus and the upper respiratory tract have a common origin from the foregut. There is increasing evidence for multiple associations of GORD with the upper respiratory tract.
\end{abstract}

Objectives To study the presence of and association of upper respiratory symptoms (URS) with GORD.

Methods Seventy adults scoring $\geq 12.5$ on a previously validated GORD symptom score (GORD patients) and 70 healthy controls who had infrequent GORD symptoms or no upper gastro-intestinal complaints completed a pre-tested URS questionnaire on the frequency of 14 URS in 5 categories (laryngeal, nasal, pharyngeal, sinusal and aural). All GORD patients underwent upper gastro-intestinal endoscopy. The calculated URS score was correlated against the GORD symptom score and endoscopy findings.

Results URS scores and individual symptom scores were higher in GORD patients compared to controls (mean $\pm \mathrm{SE}, 4.7 \pm 4.0 ; 1.9 \pm 2.3$ ). Individuals with higher GORD symptom scores reported more frequent URS. Pharyngeal symptoms had the highest correlation with the GORD symptom score $(r=0.507, p<0.001)$. The presence of oeso-phagitis did not seem to influence the frequency of reporting URS.

Conclusion Upper respiratory symptoms are common in individuals with GORD symptoms though there appears to be no association with oesophageal mucosal damage.

Ceylon Medical Journal 2016; 61: 63-67

DOI: http://doi.org/10.4038/cmj.v61i2.8301

\section{Introduction}

Gastro-oesophageal reflux disease (GORD) is the reflux of gastric contents into the oesophagus leading to oesophagitis, reflux symptoms sufficient to impair quality of life, or long-term complications [1].

The association between gastro-oesophageal reflux and lung diseases has been described from as early as 1938 [2]. In the last decade, an association between reflux disease and multiple head and neck diseases was described, including those of the larynx, pharynx, nose, sinuses and the middle ear [3]. The focus on extraoesophageal reflux disease has become pronounced over the past few years [4-7]. Most discussed are GORD associated bronchial asthma $[8,9]$, chronic cough $[10,11]$ and symptoms of the ears, nose and throat $[4,5]$. Upper respiratory symptoms (URS) have also been found to be frequent among those with symptomatic GORD with a positive oesophageal $\mathrm{pH}$ test [12]. The mechanisms proposed are, direct inhibition of upper airways and posterior nasal mucosa by gastric refluxate and/ or neurogenic inflammation triggered by oesophageal irritation [13]. Treatment of GORD has been shown to improve respiratory symptoms $[15,16]$.

Consultation for both upper gastro-intestinal and URS is common in the out-patient setting in Sri Lanka. Yet the association between them is not well documented. If an association is present, treatment of one condition could alleviate symptoms due to the other and improve overall patient management. The aim of this study was to describe the frequency of URS in patients with GORD and its association with GORD symptoms and gastroscopy findings.

\section{Methods}

Our study was a cross-sectional analytical survey using an interviewer administered questionnaire. It was conducted at the medical clinics and endoscopy unit of the University Medical Unit of the Colombo North Teaching Hospital, Ragama, Sri Lanka. The study was carried out over a period of one year from September 2010.

Departments of ${ }^{1}$ Physiology, ${ }^{2}$ Public Health, ${ }^{3}$ Pharmacology and ${ }^{4}$ Medicine, Faculty of Medicine, University of Kelaniya, Sri Lanka.

Correspondence: LA, e-mail: <lakmaliamarasiri@gmail.com>. Received 19 February and revised version accepted 8 April 2016.

This is an open-access article distributed under the terms of the Creative Commons Attribution License, which permits unrestricted use, distribution, and reproduction in any medium, provided the original author and source are credited. 
GORD patients were those individuals who scored $\geq 12.5$ on a previously validated interviewer administered GORD screening scale [17] and underwent upper gastrointestinal (UGI) endoscopy. The GORD screening scale assessed the frequency and severity of seven GORD symptoms (heartburn, regurgitation, chest pain, cough after meals, abdominal distension, difficulty in swallowing, and belching). A composite score was calculated by adding the sum of products of frequency and severity of each symptom. An individual who scored $\geq 12.5$ was considered as diagnostic of GORD. This questionnaire has been previously validated against 24-hour $\mathrm{pH}$ monitoring [17].

Upper gastrointestinal endoscopy was performed in all GORD patients. The procedure was performed by two trained physicians using a video-endoscope (Olympus, CLV-U20) and findings graded according to modified Savary-Miller criteria [19]. Additional inclusion criteria were: being between the age 15-60 years, being a nonsmoker, absence of a previous diagnosis of lung diseases or GORD (by endoscopy or $24 \mathrm{~h} \mathrm{pH}$ monitoring) and being non-pregnant.

The control group included individuals with infrequent or no upper gastro-intestinal complaints and who were not on any long term medication for treatment of upper digestive or URS and who scored $<12.5$ on the GORD screening scale. Endoscopy was not performed in controls. The same additional inclusion criteria were utilised in selection of controls.

The sample size was calculated based on a previous study [18]. In this study, the standard deviation of the upper respiratory symptom score of the patients and controls were 3.98 and 2.71 respectively and the mean score difference between patients and controls was 5.27. Based on this, using the unpaired t-test, we calculated that a minimal sample size of 67 in each group was required to have $90 \%$ power of detecting a mean score difference of 2.0 between patients and controls at a 5\% level of significance if the standard deviation in the two groups were assumed to be 4 and 3. PEPI, version 3 (Abramson $\mathrm{JH}$ and Gahlinger PM, Brixton Books, London) was used for statistical analysis. We recruited a sample of 70 participants in each category in view of possible withdrawal of participants.

The GORD patients were assessed at the endoscopy unit. Once inclusion criteria for recruitment of patients were satisfied, they were asked questions on the presence or absence of 14 upper respiratory symptoms (URS). A comparable control group, from among the patient's relatives and friends or staff of the Colombo North Teaching Hospital, was interviewed at the same time. Twenty five patients were interviewed again two days later to assess reproducibility of the questionnaire.

The frequency of 14 upper respiratory symptoms (hoarse voice, lump in throat sensation/ globus, throat clearing, cough, dryness of throat, morning sore throat, bitter taste in throat, nasal congestion, rhinorrhoea, nasal itching, purulent nasal discharge, sinus headache, ear fullness, ear discharge and earache) categorised into five groups (nasal, laryngeal, sinusal, pharyngeal and aural) were assessed with a recall period of one month, by the same interviewer.
The details of medication use for GORD and upper respiratory symptoms and relevant demographic data were recorded. Occurrence of URS were scored as 0 for absent and 1 for present with a score ranging from $0-14$. Prior to its use, the URS questions were translated in to Sinhalese and back translated. The questionnaire was pre-tested in ten people from the general population with different literacy levels to assess clarity, understandability and unambiguity of items, and ten patients presenting to the endoscopy unit with upper GI symptoms, as they would be the final target population. All participants gave informed, written consent. The study was approved by the Ethics Review Committee of the Faculty of Medicine, University of Kelaniya.

The symptoms of hoarse voice, sensation of a lump in the throat globus, throat clearing and cough were categorised as laryngeal symptoms and cough, dryness of throat, morning sore throat and bitter taste in throat were categorised as pharyngeal symptoms. The symptoms of nasal congestion, rhinorrhoea and nasal itching were categorised as nasal symptoms, purulent nasal discharge and sinus headache as sinusal symptoms, and ear fullness, ear discharge and earache as aural symptoms. Five variables (laryngeal, pharyngeal, nasal, sinusal and aural) were created indicating the presence of at least one symptom in each category. The presence of at least one upper respiratory symptom and presence of at least one symptom in each category was compared between the GORD patients and controls using non parametric tests and the Pearson Chi Square test. The association of oesophagitis with the presence of upper respiratory symptoms was assessed by Pearson Chi Square test. Binary logistic regression was used to assess the association between medication use and presence of upper respiratory symptoms. A $p$ value less than 0.05 was considered significant.

\section{Results}

A total of 70 GORD patients and 70 controls that satisfied inclusion criteria were recruited. Though some of the controls did experience infrequent GORD symptoms, all had GORD symptom scores lower than the cut-off score and were thus categorised as having a negative GORD symptom score for GORD. The GORD patients and controls had comparable baseline characteristics (Table 1). They differed significantly by the presence or absence of GORD symptoms.

The GORD patients had a significantly higher prevalence of URS compared to healthy controls (Figure 1) and also compared to those with infrequent GORD symptoms (Table 2). Heartburn, regurgitation and belching were the symptoms more commonly associated with URS.

When the URS scores were correlated with the GORD score, significant positive correlations were observed with all URS scores, with the correlation with pharyngeal symptom scores being the highest (Table 3).

Of the GORD patients, 41 had no evidence of oesophageal or gastric mucosal damage. Twenty nine had evidence of reflux oesophagitis on upper gastro-intestinal endoscopy. There was no difference in URS in those with or without oesophagitis. There was no association between of URS and medications used. 
Table 1. Demographic characteristics of participants

\begin{tabular}{|c|c|c|c|c|}
\hline & $\begin{array}{c}\text { Controls GORD } \\
\text { score } \leq 7 \\
(n=42)\end{array}$ & $\begin{array}{c}\text { Controls GORD } \\
\text { score } 7-12 \\
(n=28)\end{array}$ & $\begin{array}{c}\text { GORD patients } \\
\text { score } \geq 12.5 \\
(n=70)\end{array}$ & $\begin{array}{l}p \text { value } \\
\text { GORD }\end{array}$ \\
\hline Age in years; mean (SD) & $37.7(1.22)$ & $35.6(1.07)$ & $43.2(1.43)$ & $0.009 *$ \\
\hline Number of male: female & 17: 25 & 6: 22 & 29: 41 & $0.156^{\dagger}$ \\
\hline Body mass index & & & & \\
\hline (BMI) kg/m²; mean (SD) & $23.5(3.6)$ & 21.5 (3.5) & $23.6(3.6)$ & $0.468 *$ \\
\hline GORD score; mean (SD) & $7(0.15)$ & $10.4(1.54)$ & $45.2(2.01)$ & $0.001 *$ \\
\hline \multicolumn{5}{|l|}{ Medications used $* *$} \\
\hline $\mathrm{H}_{2}$ receptor blockers & 1 & 1 & 19 & \\
\hline Proton pump inhibitors & 1 & 3 & 60 & \\
\hline Motility drugs & 1 & 2 & 29 & \\
\hline Beta-2 agonists & 1 & 2 & 8 & \\
\hline Antihistamines & 4 & 3 & 8 & \\
\hline Oral steroids & 1 & 0 & 3 & \\
\hline Inhaled steroids & 1 & 1 & 10 & \\
\hline Methylxanthines & 1 & 0 & 4 & \\
\hline Nasal steroids & 1 & 1 & 9 & \\
\hline Antibiotics & 2 & 1 & 7 & \\
\hline
\end{tabular}

* One-way Anova ** Binary logistic regression NS ${ }^{\dagger}$ Fisher Exact test GORD - Gastro-oesophageal reflux disease

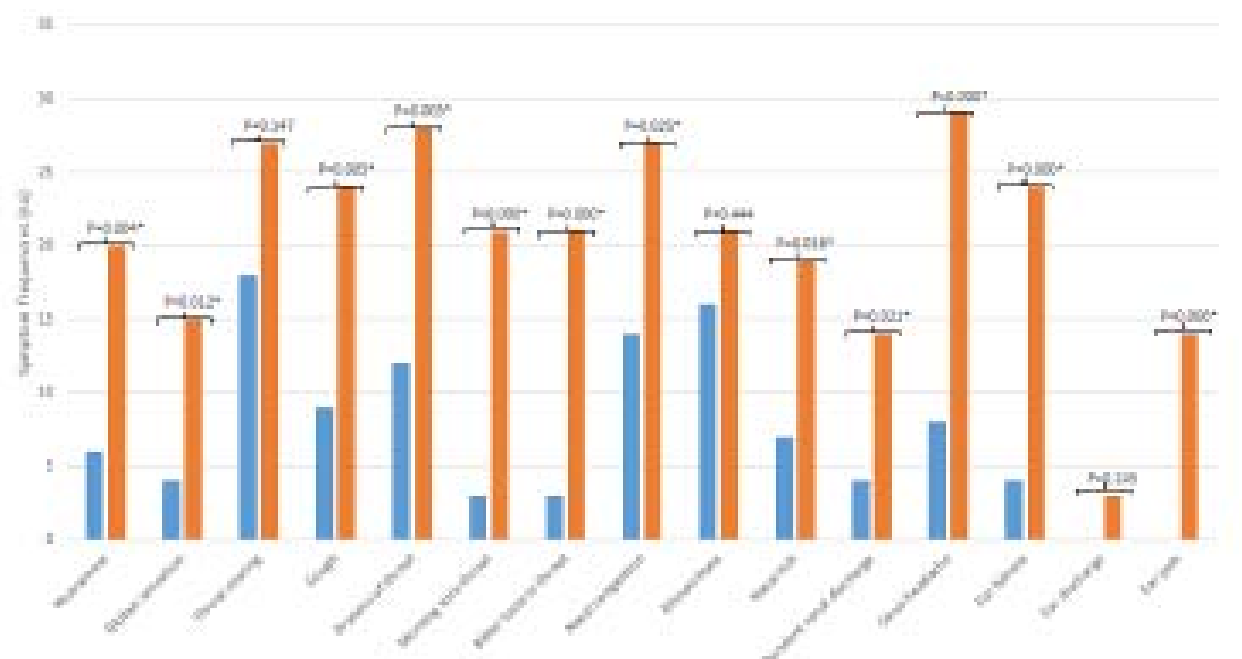

Figure 1. Upper respiratory symptom frequencies in GORD patients and controls

Table 2. URS frequencies in patients and controls with GORD symptoms

\begin{tabular}{lccc}
\hline URS & $\begin{array}{c}\text { Controls } \\
\text { GORD score } \\
(n=42)\end{array}$ & $\begin{array}{c}\text { Controls } \\
\text { GORD score } 7-12 \\
(n=28)\end{array}$ & $\begin{array}{c}\text { GORD patients } \\
\text { GORD score } \geq 12.5 \\
(n=70)\end{array}$ \\
\hline Laryngeal & $12(28.6)$ & $11(39.3)$ & $41(58.6)^{* *}$ \\
Nasal & $10(23.8)$ & $4(50.0)$ & $36(51.4)^{* *}$ \\
Sinusal & $5(11.9)$ & $9(32.1)$ & $33(47.1)^{*}$ \\
Pharyngeal & $5(11.9)$ & $1(3.6)$ & $38(54.3)^{*}$ \\
Aural & $3(7.1)$ & 2.320 .43 & $26(37.1)^{*}$ \\
Total URS score & $1.3 \pm 0.36$ & & $4.77 \pm 0.48^{*}$ \\
\hline
\end{tabular}

All values number (\%); * $p<0.001, * * p<0.05$ Pearson Chi-Square test 
Table 3. Correlation of URS scores with GORD score in all participants $(n=140)$

\begin{tabular}{ll}
\hline & $\begin{array}{c}\text { Correlation with } \\
\text { score }(\mathrm{r})\end{array}$ \\
\hline Laryngeal score & $0.361^{*}$ \\
Nasal score & $0.326^{*}$ \\
Sinusal score & $0.376^{*}$ \\
Pharyngeal score & $0.507^{*}$ \\
Aural score & $0.449 *$ \\
Total URS score & $0.512^{*}$ \\
\hline
\end{tabular}

${ }^{*} p<0.001$; Spearman rank correlation

\section{Discussion}

We assessed 14 common upper respiratory symptoms known to be associated with GORD. The symptoms were grouped as laryngeal, nasal, pharyngeal, aural and sinusal based on a previous study [18]. The study found that the frequency of URS in paticipants who experience GORD symptoms is higher compared to controls who do not experience GORD symptoms or who have infrequent GORD symptoms. Heartburn, regurgitation and belching were the symptoms more commonly associated with URS. The present study also revealed that URS were not related to endoscopic findings of GORD. GORD is known to cause oesophageal or extra-oesophageal syndromes, which can co-exist [20]. Of these, respiratory symptoms contribute to considerable morbidity and a reduction in quality of life $[11,17,21,22]$. It is thought that GORD causes respiratory symptoms by either microaspiration or reflex vagal activity.

The present study showed that of the upper respiratory symptoms, laryngeal symptoms followed by pharyngeal symptoms were the most frequent. Nasal symptoms were common too. A similar study carried out a decade ago reported that the difference in URS scores between patients and controls was mainly due to the high prevalence of laryngeal and nasal symptoms [18]. In our study, nasal symptoms also had a high correlation with the GORD symptom score. Frequent nasal symptoms have been observed in association with nasal hyper-reactivity induced by histamine provocation in GORD patients [23].

In the present study, pharyngeal symptom scores had the highest correlation with the GORD symptom score. These observations support the fact that the lower oesophagus originates from the primitive trachea [24]. The theory implicated is 'neurogenic inflammation' due to the common vagal innervations of the upper airways and the lower oesophagus. This study showed that heartburn, regurgitation and belching were the symptoms more commonly associated with URS. A previous study assessing the prevalence of GORD symptoms in asthmatics also showed that these same symptoms were the most frequent [25].
Of the 70 GORD patients, 41 showed no macroscopic evidence of oesophageal mucosal damage. It has been reported that despite the presence of symptoms, the majority of GORD patients do not have endoscopic lesions of oesophagitis. About 50\% of patients with GORD will have a normal endoscopy in referral centres but in primary care and the general population, the rate of oesophagitis is lower [26]. Further analysis showed that in GORD patients with oesophagitis, upper respiratory symptoms did not show a correlation with upper gastro-intestinal endoscopy findings. In GORD associated extra-oesophageal or atypical manifestations, endoscopic mucosal abnormalities were less common [26]. Upper gastrointestinal endoscopy was not performed in controls and we may have missed 'asymptomatic refluxers', a limitation of this study.

Medications such as beta-2 agonists, steroids and antibiotics are known to aggravate GORD and respiratory symptoms [27]. However, the present study failed to show an association between use of medications and URS.

Twenty four hour dual sensor $\mathrm{pH}$ monitoring may have detected a proportion of patients who had nonerosive reflux disease (NERD) which is a chronic, relapsing condition that can adversely affect the quality of life despite the absence of mucosal breaks at endoscopy [28]. However, the symptom score we used has good correlation with dual-sensor 24 hour $\mathrm{pH}$ monitoring variables [17]. Moreover, upper endoscopy and 24 hour $\mathrm{pH}$ monitoring are relatively insensitive for evaluating patients with atypical presentations of GORD. The ideal method would be upper airway $\mathrm{pH}$ monitoring [29]. We were unable to demonstrate proximal aspiration, and vagal function was not assessed. We could not investigate the theories implicated in pathogenesis of reflux-associated URS. However, in a previous study on 30 asthmatics we showed that genesis of respiratory symptoms was associated with mainly distal reflux. We also demonstrated that the asthmatics had a vagal hyper-reactivity and suggested that it was probable that vagal-induced reflex theory was more prominent [30]. Another study reported that there was no difference in the prevalence of URS in those with distal and proximal oesophageal acid reflux. They observed that URS are associated with GORD even in the absence of gastric acid contact with the upper airway mucosa [17]. The same study reported that the proportional increase of URS scores with reflux episodes supports an association between GORD and URS [17]. A future study incorporating ambulatory proximal and distal oesophageal and upper airway $\mathrm{pH}$ monitoring and simultaneous vagal function assessment would answer these questions.

As the GORD patients were awaiting an invasive procedure, they may have had a tendency to overrate their perception of symptoms. However, as all participants were blinded with respect to the relationship being studied, we feel that bias in reporting symptoms was minimal.

In conclusion, this study in a cohort of Sri Lankan adults confirms that URS are common in patients with 
GORD. Future studies should involve concurrent assessment of intra-oesophageal and upper airway $\mathrm{pH}$ and a therapeutic trial to assess the role of anti-acid therapy as treatment for reflux-induced URS.

\section{Conflicts of interests}

There are no conflicts of interest.

\section{References}

1. Moayyedi P, Talley NJ. Gastro-oesophageal reflux disease. Lancet 2006; 367: 2086-2100.

2. Mays EE. Intrinsic asthma in adults. Association with gastroesophageal reflux. JAMA 1976; 236: 2626-8.

3. Jecker P, Orloff LA, Mann WJ. Extraesophageal reflux and upper aerodigestive tract diseases. ORL J Otorhinolaryngol Relat Spec 2005; 67: 185-91.

4. Poelmans J, Tack J. Extraoesophageal manifestations of gastro-oesophageal reflux. Gut 2005; 54: 1492-9.

5. Richter JE. Ear, nose and throat and respiratory manifestations of gastro-esophageal reflux disease: an increasing conundrum. Eur J Gastroenterol Hepatol 2004; 16: 837-45.

6. Gislason T, Janson C, Vermeire P, et al. Respiratory symptoms and nocturnal gastroesophageal reflux: A population based study of young adults in three European countries. Chest 2002; 121: 158-63.

7. Sontag SJ. The spectrum of pulmonary symptoms due to gastroesophageal reflux. Thorac Surg Clin 2005; 15: 353-68.

8. Havemann BD, Henderson CA, El-Serag HB. The association between gastro-oesophageal reflux disease and asthma: a systematic review. Gut 2007; 56: 1654-64.

9. Sontag SJ, O’Connell S, Khandelwal S, et al. Most asthmatics have gastroesophageal reflux with or without bronchodilator therapy. Gastroenterology 1990; 99: 613-20.

10. Palombini BC, Villanova CA, Araújo E, et al. A pathogenic triad in chronic cough: asthma, postnasal drip syndrome, and gastroesophageal reflux disease. Chest 1999; 116: 279-84.

11. Harding SM, Richter JE. The role of gastroesophageal reflux in chronic cough and asthma. Chest 1997; 111: 1389-402.

12. Rosbe KW, Kenna MA, Aurebach AD. Extraesophageal reflux in pediatric patients with upper respiratory symptoms. Arch Otolaryngol Head Neck Surg 2003; 129: 1213-20.

13. Theodoropoulos DS, Lockey RF, Boyce HW, Buckantz SC. Gastroesophageal reflux and asthma: a review of pathogenesis, diagnosis and therapy. Allergy 1999; 54: 651-61.

14. Hamamoto J, Kohrogi H, Kawano O, et al. Esophageal stimulation by hydrochloric acid causes neurogenic inflammation in the airways in guinea pigs. J Appl Physiol 1997; 82: 738-45.

15. Larrain A, Carrasco E, Galleguillos F, Sepulveda R, Pope CE 2nd. Medical and surgical treatment of nonallergic asthma associated with gastroesophageal reflux. Chest 1991; 99: 1330-5.

16. Gibson PG, Henry RL, Coughlan JL. Gastro-oesophageal reflux treatment for asthma in adults and children. Cochrane Database Syst Rev 2003; 2: CD001496.
17. Amarasiri LD, Pathmeswaran A, De Silva AP, Dassanayake AS, Ranasinha CD, De Silva J. Comparison of a composite symptom score assessing both symptom frequency and severity with a score that assesses frequency alone: a preliminary study to develop a practical symptom score to detect gastro-oesophageal reflux disease in a resource-poor setting. Eur J Gastroenterol Hepatol 2010; 22: 662-8.

18. Theodoropoulos DS, Ledford DK, Lockey RF, et al. Prevalence of upper respiratory symptoms in patients with symptomatic gastroesophageal reflux disease. Am J Respir Crit Care Med 2001; 164: 72-6.

19. Savary M, Miller G, eds. The esophagus: handbook and atlas of endoscopy 1978. Switzerland: Verlag Gassman 1978.

20. Vakil N, van Zanten SV, Kahrilas P, Dent J, Jones R, Global Consensus Group. The Montreal definition and classification of gastroesophageal reflux disease: a global evidence-based consensus. Am J Gastroenterol 2006; 101: 1900-20.

21. Koufman JA. The otolaryngologic manifestations of gastroesophageal reflux disease (GORD): a clinical investigation of 225 patients using ambulatory 24-hour $\mathrm{pH}$ monitoring and an experimental investigation of the role of acid and pepsin in the development of laryngeal injury. Laryngoscope 1991; 101: 1-78.

22. Champion GL, Richter JE. Atypical presentation of gastroesophageal reflux disease: chest pain, pulmonary, and ear, nose, throat manifestations. Gastroenterologist 1993; 1: 18-33.

23. Filiaci F, Zambetti G, Luce M, Lo Vecchio A, Docimo M, Romeo R. Research of non-specific hyperractivity of upper airways in subjects with gastroesophageal reflux (G.E.R): Preliminary reports. Allergol Immunopathol (Madr) 1997; 25: 266-71.

24. Crisera CA, Connelly PR, Marmureanu AR, et al. TTF-1 and HNF-3beta in the developing tracheoesophageal fistula: further evidence for the respiratory origin of the distal esophagus. J Pediatr Surg 1999; 34: 1322-6.

25. Amarasiri LD, Pathmeswaran A, de Silva HJ, Ranasinha CD. Prevalence of gastro-oesophageal reflux disease symptoms and reflux-associated respiratory symptoms in asthma. BMC Pulm Med 2010; 10: 49-57.

26. Ronkainen J, Aro P, Storskrubb T, et al. High prevalence of gastroesophageal reflux symptoms and esophagitis with or without symptoms in the general adult Swedish population: a Kalixanda study report. Scand J Gastroenterol 2005; 40: 275-85.

27. Harding SM. Gastroesophageal reflux and asthma: insight into the association. J Allergy Clin Immunol 1999; 104: 251-9.

28. Galmiche JP. Non-erosive reflux disease and atypical gastrooesophageal reflux disease manifestations: treatment results. Drugs 2006; 66: 7-13.

29. Heidelbaugh JJ, Gill AS, Harrison RV, Nostrant TT. Atypical presentations of gastroesophageal reflux disease. Am Fam Physician 2008; 78: 483-8.

30. Amarasiri DL, Pathmeswaran A, de Silva HJ, Ranasinha CD. Response of the airways and autonomic nervous system to acid perfusion of the esophagus in patients with asthma: a laboratory study. BMC Pulm Med 2013; 13: 33-41. 